\title{
Shape and size optimisation of concrete shells
}

\author{
Antonio Tomás*, Pascual Martí \\ Department of Structures and Construction, Technical University of Cartagena, Campus Muralla del Mar, 30202 Cartagena (Murcia), Spain
}

\section{A R T I C L E I N F O}

\section{Article history:}

Received 14 October 2009

Received in revised form

16 January 2010

Accepted 1 February 2010

Available online 19 March 2010

\section{Keywords:}

Shells

Concrete

Shape optimisation

Structural response

\begin{abstract}
A B S T R A C T
Optimisation techniques may be effective in finding alternative geometries of shell structures to improve their mechanical behaviour, particularly avoiding or reducing the bending moments. This paper shows how the field of use of computers may be widened using these techniques in the design of concrete shells, allowing the user to obtain optimum designs complying with the design conditions. Some optimum geometric designs of an actual concrete shell were found, presenting an aesthetic appearance similar to that initially planned by the designer. The results confirm that significant improvements in the structural behaviour may be achieved with only slight geometric changes.
\end{abstract}

(c) 2010 Elsevier Ltd. All rights reserved.

\section{Introduction}

Triangular distribution of stresses through a cross section is uneconomic since the maximum stress occurs on the outer fibres. This is especially true for materials such as concrete, whose resistance to tension is small compared to compression, and the strength capacity of the cross section is drained as soon as the minimum value is reached.

The structural behaviour of shells, compared to that of other types of structures, is characterized by a higher mechanical efficiency. Concrete shells depend on their configuration, not on their mass, for stability. If appropriate designs are carried out, shells can support high loads and allow one to cover important spaces using little material and/or thickness. Moreover, shells present an attractive lightness and elegance from an aesthetic point of view, leading some authors to refer to them as the "structural elegance" [1], the "prima donna" of structures [2] or that structure in which one discovers what is resisting only contemplating its shape [3].

The structural behaviour of shells is developed essentially due to their form. It would be interesting to find, if possible, small modifications in their geometry without modifying their initial aesthetic configuration too much and still complying with the design conditions. These modifications would improve that mechanical behaviour still further. It could be attempted, for example, to reach a distribution of stresses in the thickness which is as uniform as possible, and this would imply to have shells free of bending or, at least, with some acceptable bending values [4].

\footnotetext{
* Corresponding author.

E-mail address: antonio.tomas@upct.es (A. Tomás).
}

Among different techniques used in form-finding of concrete shells [5,6], optimisation techniques represent an effective means to achieve this purpose in the field of computational mechanics (see $[7,8]$ among others). The development of optimisation techniques was strongly boosted by the tremendous increase in computational and graphical capacities. These techniques permit to obtain alternative geometric forms of shells and improve their mechanical behaviour, complying with the design conditions (stress constraints, construction conditions, etc.) in an optimum way (minimum weight, maximum stiffness, minimum stress level, etc.).

In this paper, optimisation techniques are applied to find optimum geometric designs of concrete shells complying with the design conditions. Some optimum geometric designs of an actual concrete shell have also been found, being close to a preconceived design, i.e. the resulting geometries should have an aesthetic shape similar to the form of the initially designed structure, which is a hyperbolic paraboloid (hypar). Slight changes in the form of these types of structures can introduce important improvements in their mechanical behaviour (see [9-11] among others). To achieve this task, different objective functions can be used, such as the strain energy, the weight of the structure, or the tensile stress in both faces of the shell. The parameters that govern the geometry and the thickness of the shell were used as variables. The constraints were the minimum thickness, the tensile stresses in concrete, and several parameters of geometric control.

\section{Shape optimisation of concrete shells}

The design process of the membrane state stresses in a shell structure can be hindered by a series of factors such as the 
application of concentrated loads, the existence of free edge boundary conditions or the possible incompatibility that could appear between a given form and the thickness of the shell. Since these factors are strongly dependent on the form of the structure, it becomes essential to incorporate, in the design process, shape optimisation techniques that account for them. Therefore, the main objective in shell design would be to find a form that, considering the specific properties of the material, satisfies the following design conditions [1]:

(1) stresses and displacements enclosed within an interval;

(2) membrane stress state (or a close approximation of it);

(3) boundary conditions met for all load configurations;

(4) buckling instability phenomena avoided;

(5) sensitivity of the structural response to possible variations in the geometry minimised; and

(6) a final shape that meets some aesthetic criteria.

The aim of the optimal structural design is to obtain a design, a set of values for the design variables, which minimises an objective function and complies with the constraints that depend on the variables.

The design variables of a structure can be properties of the cross-section of the elements (surface areas, thicknesses, inertia moments, etc.); structural geometry parameters; structural topology parameters (element densities in the range from 0 to 1 ) [12]; and properties of the material of the structure. Traditionally, the weight of the structure is the most common objective function used. Nevertheless, the weight is not the determining factor in other applications, and other objective functions such as cost, reliability, stiffness, etc. are used. The constraints are the conditions that the design must comply with in order to be regarded as valid.

The optimum design problem was formulated as follows:

To find the variable vector of design $\mathbf{x}$ which

minimises $f(\mathbf{x})$

subject to $\quad h_{j}(\mathbf{x})=0 \quad j=1,2, \ldots, m_{i}$

$$
\begin{array}{ll}
g_{k}(\mathbf{x}) \geq 0 & k=1,2, \ldots, m_{d} \\
x_{i}^{L} \leq x_{i} \leq x_{i}^{U} & i=1,2, \ldots, n
\end{array}
$$

where $\mathbf{x}$ is the $n$-dimensional vector of the design variables; $f(\mathbf{x})$ is the objective function; $h_{j}(\mathbf{x})$ is the $j$ th equality design constraint; $g_{k}(\mathbf{x})$ is the $k$ th inequality design constraint; $m_{i}$ is the number of equality constraints; $m_{d}$ is the number of inequality constraints; $n$ is the number of variables; and $x_{i}^{L}\left(x_{i}^{U}\right)$ is the lower limit (upper limit) of the variable $i$ (e.g. [13] among others).

This problem was solved by mathematical programming using the optimisation module in ANSYS [14], which has a conventional first-order method using the first derivatives of the objective function and constraints with respect to the design variables. The module converts the optimisation problem with constraints into an unconstrained problem by adding penalty functions to the objective function. For each iteration, gradient calculations, which employ a steepest descent or conjugate direction method, are performed to determine a search direction. A line search strategy is adopted to minimise the unconstrained optimisation problem.

Depending on the type of design variables to consider, one of three optimisation methodologies are usually used in the formfinding process: (i) using the coordinates of the nodes of the FE mesh; (ii) defining a parametric model using some variables of geometry; (iii) and stating a set of predefined geometries with the optimum shape being a lineal combination of them with the design variables as combination factors [15]. The second of these methodologies is used in this paper.

Several authors have proposed suitable objective functions to optimise the shape of shells [1,16-18]. For example, in order to find a state of membrane stresses, the highest principal tensile stress can be used as objective function. An alternative way is to substitute this condition for a constraint to avoid tensions in all points of the shell.

To improve the behaviour of the structure in case of instability phenomena, the buckling load can be used as objective function. As the response of shell structures is very sensitive to geometric imperfections, it is recommended to include the latter function in the maximisation process of the buckling load. A first approach of the buckling load can be obtained by analysing the initial stability through an eigenvalue analysis. However, in order to obtain a more realistic value, nonlinear analysis is needed.

To minimise displacements in the whole structure, a function called "Volumetric Displacement" (VD) may be defined according to the following expression [19]

$V D=\sum_{i=1}^{n} d_{i} \times S_{i} \times$ Thick $_{i}$

where $d_{i}$ is the displacement vector modulus at each point $i ; S_{i}$ is the area of influence at this point; and Thick $k_{i}$ is the average thickness of the structure at the mentioned area. With respect to the maximum displacement technique, the advantage of the $V D$ function is that it provides a wider view of displacements throughout the structure.

To reduce the bending in the shell, it is appropriate to minimise the strain energy $S_{E}$, or equivalently, to maximise the stiffness of the structure

$S_{E}=\frac{1}{2} \int \sigma \varepsilon \mathrm{d} V$

where $\sigma$ are the stresses and $\varepsilon$ the strains, both calculated in all points of the shell. Minimising the strain energy leads to lower stresses and deformations in the shell, but in an implicit way, it allows one to meet the mentioned objectives simultaneously. In other words, the behaviour of the structure is improved together with (i) a higher buckling load and (ii) a "relaxed" state of stresses close to the membrane state.

Considering that all the design conditions stated above can easily be satisfied by increasing the weight of the shell, it could be convenient in some cases to limit the weight to a maximum value, or even to introduce it in the optimisation process as equality constraint.

Since a distortion may appear in the geometry of the structure in such a way that its shape differs too much from the preconceived form, and from the desired aesthetic criteria, the inequality constraints $g_{k}(\mathbf{x})$, which are commonly used to limit the tensile stresses and the displacements, can also be necessary to limit some parameters of shape control.

The inequality constraints $g_{k}(\mathbf{x})$ are commonly used to limit the tensile stresses and the displacements. However, a distortion may appear in the geometry of the structure leading to a shape that differs too much from the preconceived form and the desired aesthetic criteria. In this case, constraining some parameters of shape control can also be necessary. For example, in the actual shell used in Section 4 two shape parameters of the shell are restricted: the height of the highest point of the free edge, and the radius or distance from the $Z$-axis to the support.

\section{Example: Shape optimisation of a shell with different boundary conditions}

The case of shape optimisation of a concrete shell used by Bletzinger and Ramm [17] is studied. It is subjected to its own weight and a vertical uniform load, for different design criteria. The study has been enlarged in this case for different boundary conditions. The shell thickness is $50 \mathrm{~mm}$ and the structure covers 


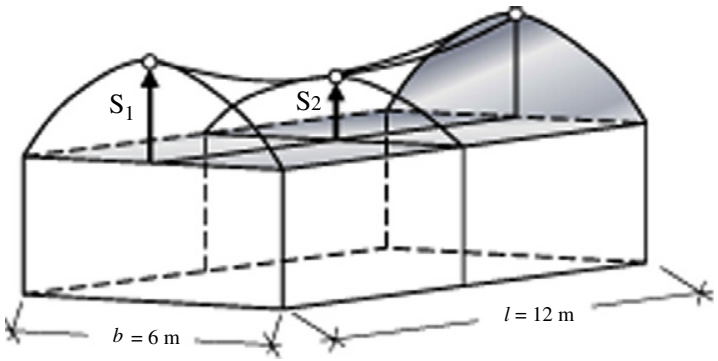

Fig. 1. Optimisation model.

a surface of $6 \times 12 \mathrm{~m}$. Young's module of the material is $30 \mathrm{GPa}$ and Poisson's module is 0.2 . The structure is subjected to a vertical uniform load of $5 \mathrm{kN} / \mathrm{m}^{2}$. The design variables are $S_{1}$ and $S_{2}$ (Fig. 1 ), with both adopting a value of $3 \mathrm{~m}$ in the initial model. The shell can be supported on the right edges, on the curved ones or on them all at the same time (Fig. 2).

The optimisation of just a quarter of a shell has been carried out applying symmetry conditions, under three different objective functions: weight, strain energy or stress levelling. For the weight, the tensile stresses have been constrained to $2 \mathrm{MPa}$ and the compressive ones to $-20 \mathrm{MPa}$. The second objective function is the sum of the strain energy in the elements of the model, defined by Eq. (3). In this case, it is irrelevant to adopt any constraint for the tensile stresses, because they remain under the strength of the material along the runs. The stress levelling $S L$ is a function used by Bletzinger and Ramm [17] and can be redefined, expressed in a discrete way for the elements of the mesh, as

$S L=\sum_{i, j}\left(\sigma_{i j}-\sigma_{a}\right)^{2} \quad i=1,2,3, j=1,2, \ldots, n$ where $\sigma_{i j}$ is the average principal stress $i$ at the nodes of the element $j ; n$ is the number of elements in the mesh of the model; and $\sigma_{a}=f_{t}$ is the tensile strength of the material.

In this case, it is also irrelevant to adopt any constraint for the tensile stresses. The results of the optimisation runs are shown in Fig. 2.

$W=$ weight $; \quad S E=$ strain energy;

$S L=$ stress levelling; $\quad$ units of $S_{1}, S_{2}$ are (m).

In case the shell is supported on the right edges, when minimising the weight, the shape shows a trend toward the plane form. The weight has decreased $32.01 \%$. Using the strain energy or the stress levelling as objective function, the geometry adopts the form of a hyperbolic paraboloid, changing from simple to negative double curvature, and obtaining an important decrease in the objective function of $88.67 \%$ and $96.69 \%$, respectively.

In case the shell is supported on the curved edges, when minimising the strain energy or the stress levelling, the geometry adopts the shape of a hyperbolic paraboloid again, but this time in a more marked way because of the increasing of the variable $S_{1}$ and of $S_{2}$ adopting a value close to zero, which causes the shell to "hang" from the curved edges. The fall in the strain energy is again important, $97.00 \%$, while it remains very similar in the stress levelling, 96.61\%. Using the weight as objective function the geometry also tends toward the hyperbolic-parabolic form, although the decrease in the weight is smaller than before, being just $6.55 \%$.

Lastly, in case the shell is supported on all the edges, when minimising the strain energy or the stress levelling, the geometry changes again from simple to double curvature, but this time it is positive, which causes a "bulging" of the central area. The fall in the strain energy is $54.98 \%$, and $64.01 \%$ in the stress levelling, the smallest values in the three cases. Using the weight as objective function, the geometry changes from a parabolic cylinder to an

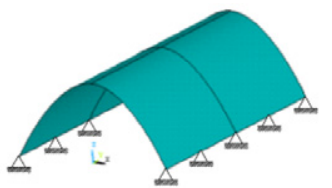

Initial

$W=33.11 \mathrm{kN}$

$S E=2675.50 \mathrm{kNm}$ $S L=2670.40 \mathrm{kN}^{2} / \mathrm{m}^{4}$

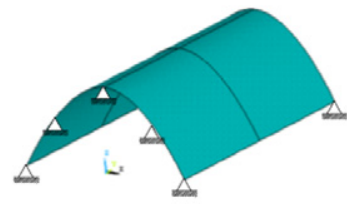

Initial

$W=33.11 \mathrm{kN}$

$S E=9935.20 \mathrm{kNm}$

$S L=287.70 \mathrm{kN}^{2} / \mathrm{m}^{4}$

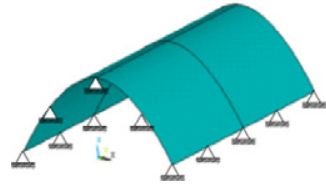

Initial

$W=33.11 \mathrm{kN}$

$S E=1036.00 \mathrm{kNm}$

$S L=571.21 \mathrm{kN}^{2} / \mathrm{m}^{4}$

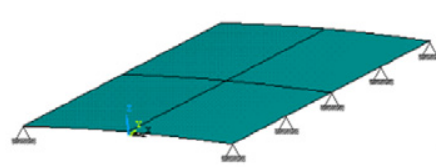

Objective function $W$ $W=22.51 \mathrm{kN}$ $S_{1}=0.06 S_{2}=0.08$

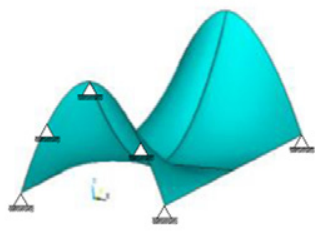

Objective function $W$ $W=30.94 \mathrm{kN}$ $S_{1}=4.57 \quad S_{2}=0.01$

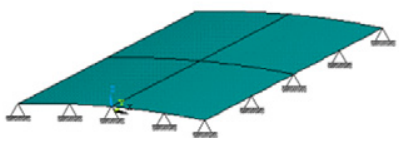

Objective function $W$ $W=22.54 \mathrm{kN}$ $S_{1}=0.14 \quad S_{2}=0.16$

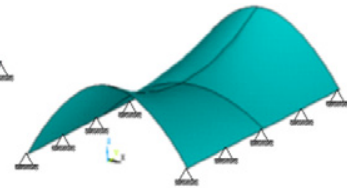

Objective function $S E$ $S E=303.01 \mathrm{kNm}$ $S_{1}=2.67 S_{2}=1.36$

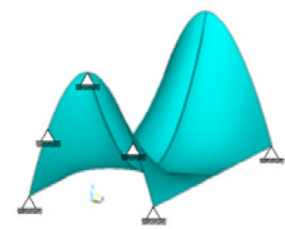

Objective function $S E$ $S E=298.40 \mathrm{kNm}$ $S_{1}=5.80 S_{2}=0.01$

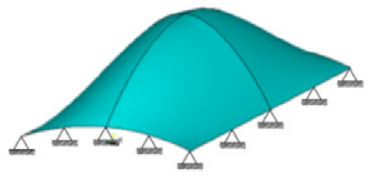

Objective function $S E$ $S E=466.40 \mathrm{kNm}$ $S_{1}=0.55 S_{2}=2.92$

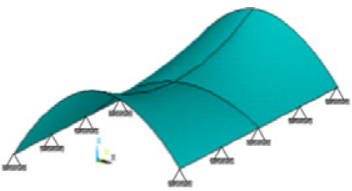

Objective function $S L$ $S L=88.48 \mathrm{kN}^{2} / \mathrm{m}^{4}$ $S_{1}=2.39 S_{2}=1.31$

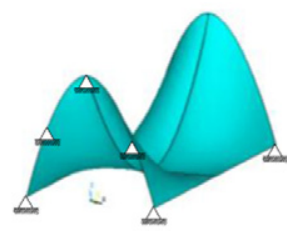

Objective function $S L$ $S L=9.76 \mathrm{kN}^{2} / \mathrm{m}^{4}$ $S_{1}=5.50 \quad S_{2}=0.01$

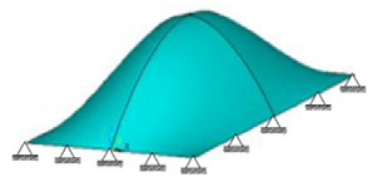

Objective function $S L$ $S L=205.56 \mathrm{kN}^{2} / \mathrm{m}^{4}$ $S_{1}=0.01 \quad S_{2}=3.11$

Fig. 2. Shell with different boundary conditions. Results of the optimisation runs 
almost plane form, decreasing its value $31.92 \%$, very similar to the first case, as indeed it had to be since the final geometries are almost identical.

After these results, it can be summarised that using the strain energy or the stress levelling as objective functions, the geometry of the shell tends to forms of double curvature, with a marked decrease in the tensile stresses. In the case of the weight, the geometry tends to plane forms, slab type, with the surface minimising, since the thickness remains constant. This latter objective function is usually used in the field of structural optimisation, although it is interesting to keep others in mind such as the strain energy, because of the rational way of improving the mechanical behaviour of a shell, since the tensile stresses and the deformations of the structure decrease simultaneously.

Although shells can adopt any form, double-curvature shells present important advantages in their mechanical behaviour compared to other forms [20-24], since it is possible to avoid the appearance of bending moments in them. Their particular behaviour is due to the arch-effect in two planes and, in contrast to the arch contained in only one plane, it allows supporting different load configurations, mainly by means of membrane internal forces, with a very low risk of bending. Moreover, these surfaces have a practically unalterable form, and are in equilibrium whatever the type of distribution of the loads, within certain limits. This geometry involves enough mechanical features to be a very efficient structure, even without stiffening elements such as edge beams. This implies that shell structures designed to behave as membranes are, by themselves, optimum structures.

Unfortunately, as usually occurs in optimum systems, this high mechanical efficiency induces a structural behaviour that is extremely sensitive to imperfections (see how the buckling load of a shell decreases when slight geometric imperfections appear [9-11]). Considering the normal construction procedures, the probability that local defects exist in the geometry or thickness, or that other constructive problems arise (too young concrete, scaffolding removed defectively, etc.), is relatively high. Some real examples associated to these problems may stand out, such as the frequent appearance of cracks when removing scaffolding under the shell. A more serious case was the partial collapse of the chapel of Lomas de Cuernavaca [25]. Other cases may be consulted in the literature [26-28].

Since the structural behaviour of shells is basically due to their form, it is logical to think of the possibility of finding small changes in the geometry that, without distorting the initial aesthetic configuration too much, improve that mechanical behaviour even further, whilst complying with the design conditions. For example, it could be attempted to achieve a stress distribution along the thickness being the most uniform possible, which would imply to have free-bending shells or, at least, with some acceptable bending (tensile stresses) values. The improvement of this mechanical behaviour through shape optimisation is suggested by Ramm [6]. By using shape optimisation, more quality in the design process is achieved, since it helps to obtain structures of quasi-perfect behaviour.

\section{Example: Shape and size optimisation of an actual hypar}

The shape and size optimisation of an actual concrete shell structure is presented below. It was designed for the entrance of the Universal Oceanographic Park in Valencia, Spain (Fig. 3). The roof shell is based on hand-drawn sketches by Felix Candela, which inspired the subsequent making of the building project. This structure is the posthumous work of Candela. The geometry of the structure consists of the intersection of three lobes whose midsurfaces describe the shape of a hypar. The basic parameters and design variables used in the definition of the geometry are shown a

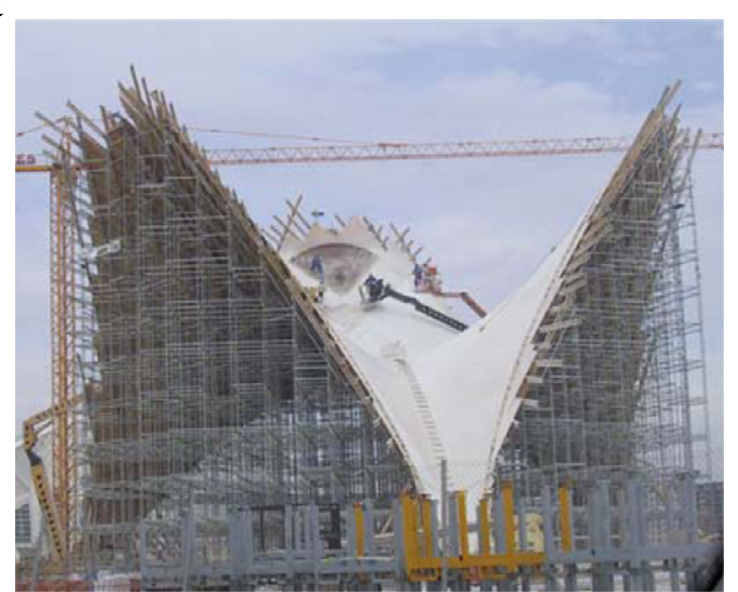

b

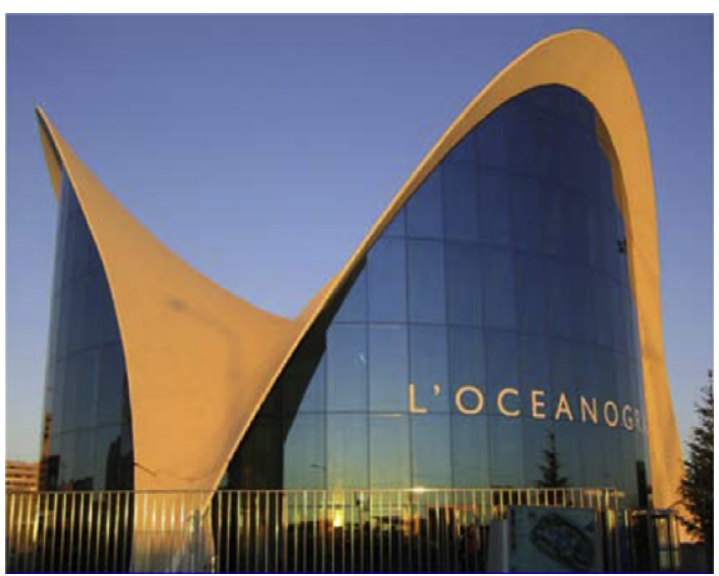

Fig. 3. Shell structure at the entrance of the Universal Oceanographic Park (Valencia, Spain). (a) The shell under construction. (b) The shell today.

in Sections 4.3.2 and 4.3.3. A complete description of that definition may be consulted in [29]. After analysing the results of the initial design, several optimisation processes under predominantly gravitational loads were carried out.

\subsection{CAD model}

To generate the CAD model, a global system of cylindrical coordinates with origin at the point of intersection of the three paraboloids was defined. The patches defining the mid-surface of the structure were derived from the coordinate system. The ANSYS program of finite elements was used to model the shell.

The following sequence of operations was programmed:

- obtain a set of points (keypoints) contained in the mid-surface,

- link the points by curved lines (splines), and

- obtain ruled surfaces (Coons patches) by the splines to generate the geometry of the structure.

Overall, there are 1945 keypoints, which, linked in sets of six, create 432 splines, which generated 216 Coons patches forming the model (Fig. 4).

In addition, the shell has been stiffened by two types of ribs with similar dimensions as used by Domingo, Lázaro y Serna [23]. One type spreads from the support to the centre of the structure (main ribs). The other one surrounds a small central hole made in the shell 
a

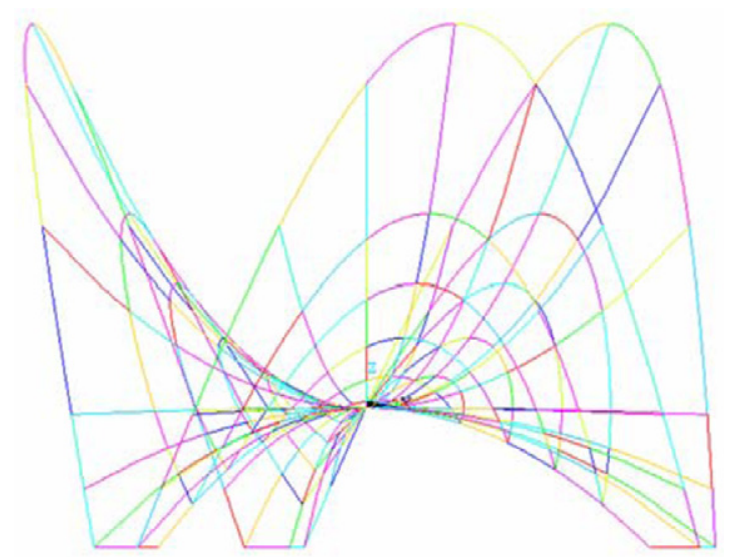

b

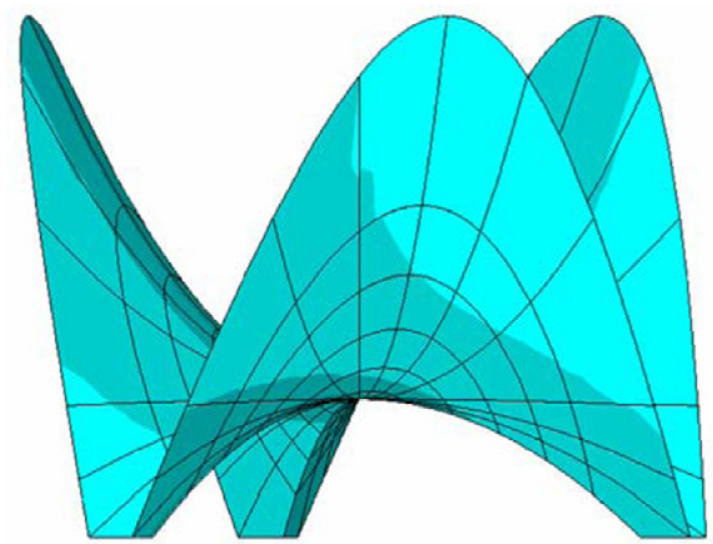

Fig. 4. CAD model of the structure. (a) Splines. (b) Coons patches.

to avoid problems when meshing, gauging a band of $200 \mathrm{~mm}$ wide (hole rib).

The implementation of the CAD model in ANSYS was done by enabling the form of the structure to be modified by varying the values of some design parameters (thicknesses of the elements that form the structure and those to control the geometry).

\subsection{Analysis model}

Due to the symmetry of the structure, the different analyses were carried out on one sixth of the shell. Prior to meshing the surface of the CAD model, the thickness, material, element type and geometric features of the elements were defined. The mesh has 4375 elements. It was necessary to create a small circular hole of $100 \mathrm{~mm}$ diameter at the intersection of the lobes, in order to avoid meshing problems arising from the distortion of the elements generated in the area surrounding the centre, which have very acute angles (Fig. 5).

The material of the structure is concrete. The mid-surface of the shell was provided with reinforcement netting, which was used to account for time-dependent effects of the concrete, since these effects can have a considerable influence when the thickness of the shell is small with respect to other dimensions. Therefore, the contribution of the reinforcement was not considered in the analysis, except in the effects of its density. The specific weight of the material is $25.00 \mathrm{kN} / \mathrm{m}^{3}$ (a value commonly used for a

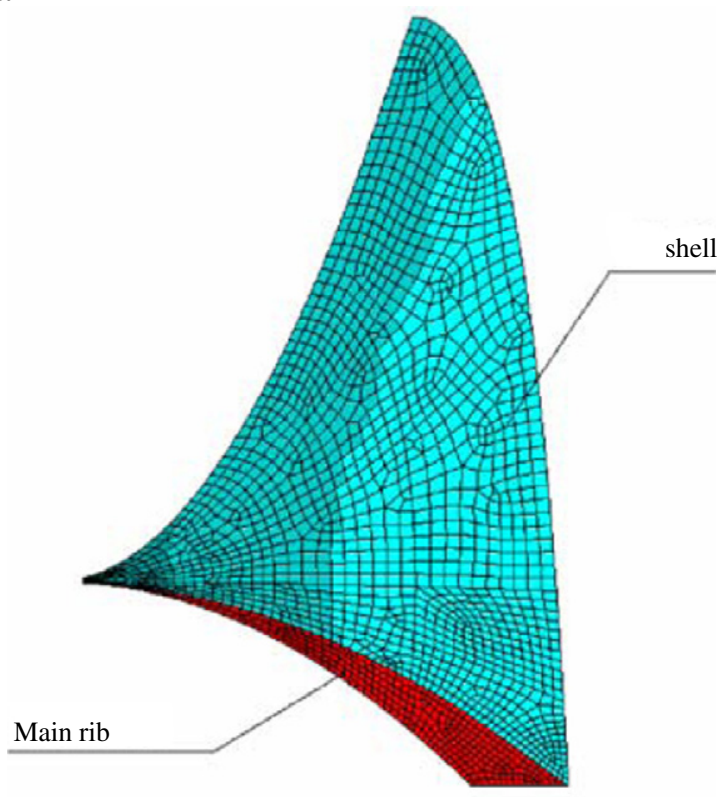

b

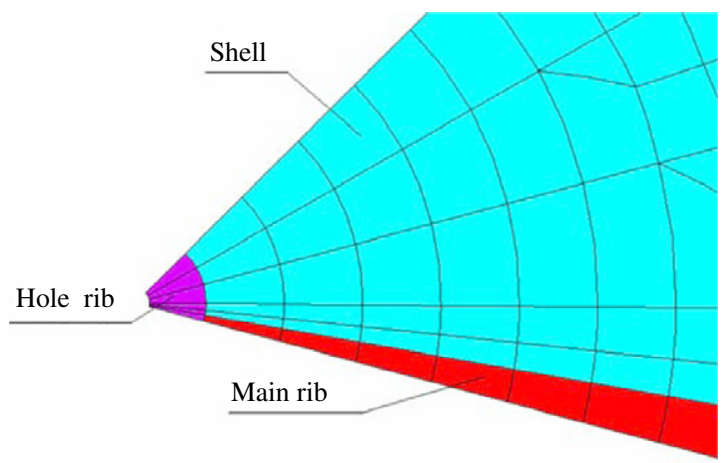

Fig. 5. FE mesh. (a) One sixth of the structure. (b) Enlarged detail of the lobes intersection area.

reinforced concrete). The mechanical properties are $30.00 \mathrm{MPa}$ for the characteristic compressive strength of concrete $\left(f_{c k}\right), 20.00 \mathrm{MPa}$ for the design compressive strength $\left(f_{c d}\right), 1.35 \mathrm{MPa}$ for the design tensile strength $\left(f_{c t, d}\right), 0.20$ for Poisson's ratio, and $28576 \mathrm{MPa}$ for the secant Young's modulus referred to the concrete age of 28 days.

There are essentially two different ways of formulating the elements used in concrete shells: those based on the degenerate solid approach and those based on a shell theory [30]. An element belonging to the second type was used, the Shell93 in ANSYS [31]. The element has six degrees of freedom at each node: translations in the nodal $x, y$, and $z$ directions and rotations about the nodal $x, y$, and $z$-axes. The deformation shapes are quadratic in both in-plane directions. The element has plasticity, stress stiffening, large deflection, and large strain capabilities. The material property matrix for the element includes a formulation to avoid shear locking.

Because of the symmetry of geometry and loads, the analysis of a lobe with an angle of $60^{\circ}$ was carried out, applying symmetry boundary conditions to the nodes in the symmetry planes, and 
restricting the translations in the $x, y$, and $z$ directions of the nodes in the foundation plane.

The applied loads are the weight of the structure and the distributed load of $1 \mathrm{kN} / \mathrm{m}^{2}$. The action of the wind was not considered because of its slight contribution to the whole load, only $5.87 \%$ of the gravitational loads. This percentage is a maximum value obtained by adopting a simplified and safe hypothesis for introducing wind into the analysis model [29].

\subsection{Formulation of the optimum design problem of the hypar shell}

\subsubsection{Objective functions}

The objective functions were the following:

(1) strain energy of the structure,

(2) weight of the hypar, and

(3) highest tensile stress at the nodes of the model.

\subsubsection{Design variables}

The concrete shell structure under study consists of the intersection of three hyperbolic paraboloids. Each paraboloid contains two sets of generating lines, each of these sets parallel to a director plane. The intersection of the two director planes defines the $Z$-axis, forming together an angle $\omega$. The expression that defines the mid-surface of the shell in cylindrical coordinate system is given by:

$$
\begin{aligned}
z= & K r^{2}\left[\cos \left(\frac{90+\omega}{2}-\alpha\right)-\frac{\sin \left(\frac{90+\omega}{2}-\alpha\right)}{\tan (\omega)}\right] \\
& \times\left[\cos \left(\alpha-\frac{90-\omega}{2}\right)-\frac{\sin \left(\alpha-\frac{90-\omega}{2}\right)}{\tan (\omega)}\right]
\end{aligned}
$$

where $K$ is the mid-surface constant, $\omega$ the angle between the two director planes of the hypar and $z, r, \alpha$ are the cylindrical coordinates.

The free edge of each hypar is defined by an inclined plane containing the line joining the points R2 and R2' and forms an angle $\beta$ with respect to a horizontal plane (Fig. 6).

The following design variables, whose initial values were proposed in sketches by Candela, were used for the design of the entrance to the Oceanographic Park:

$K$ Mid-surface constant. In the optimisation processes, the initial value was $0.14 \mathrm{~m}^{-1}$, with $0.13 \mathrm{~m}^{-1}$ and $0.17 \mathrm{~m}^{-1}$ being the minimum and maximum values, respectively.

$\omega$ Angle between the two director planes. Its initial value is $90^{\circ}$ (equilateral hypar). The stated lower and upper limits are $84^{\circ}$ and $91^{\circ}$, respectively.

$\beta$ Angle of the inclined plane. Its initial value was $75^{\circ}$, allowing for a variation interval between $74^{\circ}$ and $75^{\circ}$, since the design is very sensitive to this variable. With this interval, the structure cannot be lower than $19 \mathrm{~m}$ in height.

$e_{1}$ Shell thickness. A minimum initial value of $60 \mathrm{~mm}$ for constructive conditions was chosen. In the optimisation processes, the thickness was allowed to range from 60 to $80 \mathrm{~mm}$.

$e_{2}$ Hole rib thickness. The initial value was $80 \mathrm{~mm}$, with a minimum of $60 \mathrm{~mm}$.

$e_{3}$ Main ribs thickness. The initial value was $350 \mathrm{~mm}$, with a variation interval between 60 and $400 \mathrm{~mm}$.
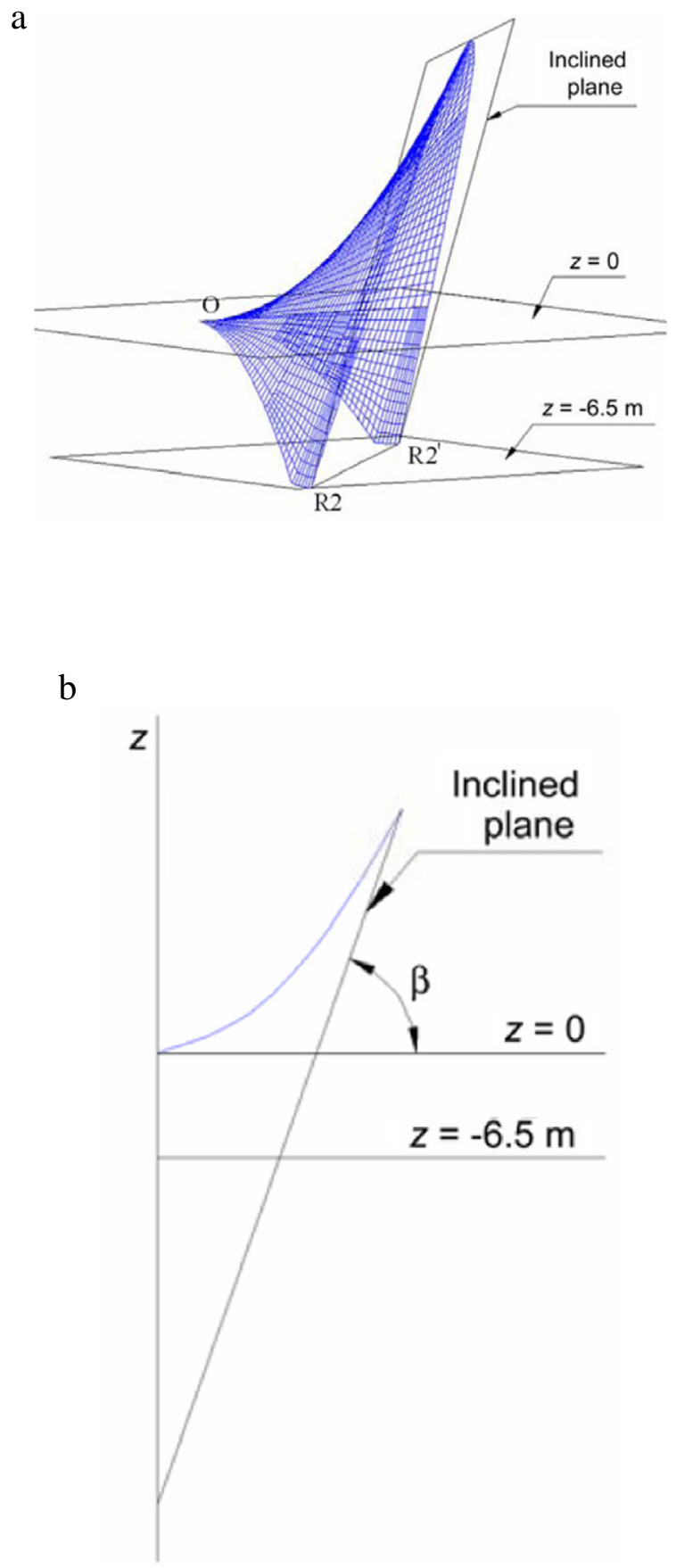

Fig. 6. Inclined plane sectioning the hypar to define the free edge of a lobe. (a) Perspective. (b) Side view.

\subsubsection{Constraints}

The maximum extreme-fibre stresses at the outer surfaces of the shell were restricted depending on the design strength of the material of the shell:

$\sigma_{t} \leq f_{c t, d}$

$\sigma_{c} \leq 0.85 f_{c d}$

where $\sigma_{t}$ is the tensile stress and $\sigma_{c}$ the compressive stress. Two shape parameters of the hypar (the height of the highest point of the free edge and the radius or distance from the $Z$ axis to the support) were also restricted. This was necessary because the values of these parameters tend to decrease during the optimisation runs, distorting the geometry of the structure 
Table 1

Optimisation processes. Final values of variables of geometry.

\begin{tabular}{|c|c|c|c|c|c|c|c|}
\hline Process & $t_{1}(\mathrm{~mm})$ & $t_{2}(\mathrm{~mm})$ & $t_{3}(\mathrm{~mm})$ & $K\left(\mathrm{~m}^{-1}\right)$ & $\omega\left(^{\circ}\right)$ & Radius (m) & Height $(\mathrm{m})$ \\
\hline Initial model & 60.0 & 80.0 & 350.0 & 0.140 & 90.00 & 13.63 & 24.39 \\
\hline$S E\left(t_{\min }=60 \mathrm{~mm}\right)$ & 61.2 & 60.0 & 362.1 & 0.158 & 85.42 & 11.87 & 19.04 \\
\hline$S E\left(t_{\min }=80 \mathrm{~mm}\right)$ & 80.0 & 95.4 & 400.0 & 0.165 & 84.98 & 11.54 & 19.00 \\
\hline$W\left(t_{\min }=60 \mathrm{~mm}\right)$ & 60.0 & 71.9 & 264.3 & 0.150 & 85.87 & 12.27 & 19.02 \\
\hline$W\left(t_{\min }=80 \mathrm{~mm}\right)$ & 80.0 & 81.0 & 333.1 & 0.141 & 86.45 & 12.79 & 19.05 \\
\hline$\sigma_{t}\left(t_{\min }=60 \mathrm{~mm}\right)$ & 159.4 & 80.9 & 384.6 & 0.139 & 86.53 & 12.91 & 19.01 \\
\hline
\end{tabular}

$S E=$ strain energy; $W=$ weight; $\sigma_{t}=$ tensile stress; $t_{\min }=$ minimum thickness.

and thus departing significantly from the hyperbolic paraboloid. Furthermore, its appearance would not match the design criteria. The stated minimum values are $19 \mathrm{~m}$ for the height of the free edge and $11.5 \mathrm{~m}$ for the radius.

\subsection{Results}

In the first stage, the analysis of the initial design allowed to obtain outstanding information, such as the stresses and displacements at the points of the structure and the buckling load. The initial design was developed starting from the hand-drawn sketches by Candela for the Oceanographic Park.

In the second stage, several optimisation runs were executed with the purpose of improving the structural behaviour under the worst of several load combinations. The optimisation runs of the initial model were classified into two groups depending on:

(1) The objective function used (strain energy, weight or tensile stress), and

(2) the minimum thickness allowed (60 or $80 \mathrm{~mm}$ ).

For each objective function, two optimisation runs were carried out depending on the minimum thickness allowed.

A buckling study of the structure was carried out for the initial model with three different thicknesses and for the optimum designs. Two types of analysis have been used (linear and nonlinear).

Following the recommendations of Lee and Hinton [32] for this kind of shape optimisation processes with linear analysis, the behaviour of the shell against instability has been studied using nonlinear analysis.

Moreover, it was considered of interest to calculate the final values of two geometric parameters: (i) the height of the free edge of the hypar and (ii) the radius or distance in ground plan from the centre of the structure to one of its supports. The comparison of these parameters in the different processes could help in visualizing and showing the changes that have taken place in the geometry of the initial model. The final values of the variables of geometry in the different optimisation processes are shown in Table 1.

It is observed that the angle $\omega$ (angle between the director planes) decreases in all the optimisation runs, implying that the hypar is no longer equilateral. On the other hand, the height of the initial model decreases in all runs tending to the stated minimum value of $19 \mathrm{~m}$. Regarding the thickness of the shell, it can be seen that the allowed minimum value is reached when the strain energy and the weight are optimised. However, when the maximum tensile stress is optimised, the thickness of the shell is nearly $160 \mathrm{~mm}$, indicating the high cost of a form having the membrane behaviour when geometric constraints are used.

The final values of the objective functions are shown in Table 2 , together with three additional parameters whose analysis and comparison could be useful: the shell thickness $e_{1}$, the maximum compressive stress $\sigma_{c, \max }$ and the maximum vertical displacement $U_{z, \max }$.

From the above results, it could be seen that in all the optimisation processes, the maximum compressive stresses are below $5 \mathrm{MPa}$ and the maximum tensile stresses are lower than the design

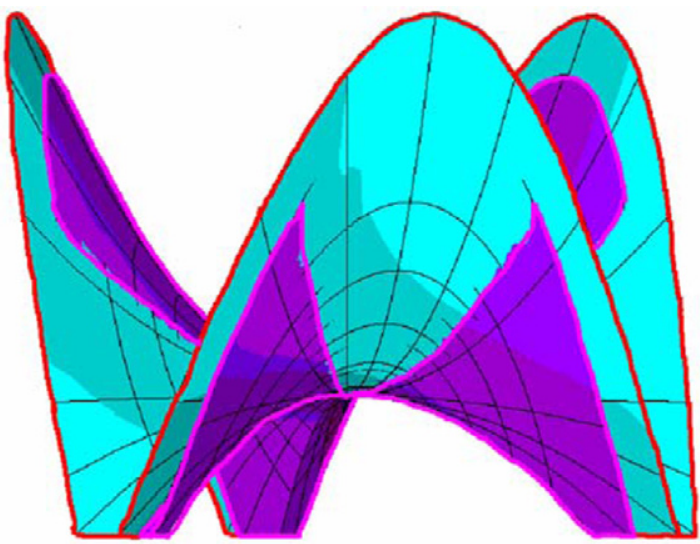

Fig. 7. Intersection of initial model (light shaded) and a final design (dark shaded)

tensile strength of the concrete. In addition, the maximum vertical displacement of the structure is lower than $4 \mathrm{~mm}$, which concurs with the results obtained by Ortega and Arias [21] for this type of optimised structures, where vertical displacements are below $10 \%$ of the shell thickness.

When tensile stress is the objective function, the weight approximately doubles the values obtained in the other optimisation processes. However, no substantial decrease in the tensile stress is achieved, which confirms the high cost of a form with a membrane behaviour using geometric constraints, as mentioned previously.

The geometries of the initial design and of one of the final designs are compared in Fig. 7. The final design was obtained using strain energy as the objective function and a minimum thickness of $60 \mathrm{~mm}$ as the constraint. Both geometries are intersected to provide a better perspective and show the slight differences between them.

Finally, Table 3 shows the results of a buckling analysis by using: eigenvalue (linear) analysis; nonlinear analysis considering geometric and material nonlinearity; and the latter analysis but modified by a factor $\left(\alpha_{2}\right)$ to consider concrete creep according to IASS Recommendations [33]. The buckling loads correspond to the final designs in each optimisation process and to the initial model with a thickness of $60 \mathrm{~mm}$.

From the stability study, the high buckling load obtained shows the high stiffness of this type of structures. This stiffness is also confirmed by the maximum vertical displacement obtained of $8.73 \mathrm{~mm}$ in the initial model, and less than $4 \mathrm{~mm}$ in the optimum designs (Table 2 ).

The influence of the thickness is decisive, because its increase implies a decrease of the shell slenderness and therefore an increment in the buckling load.

Secondly, the buckling load is approximately double in the optimum designs with respect to the initial models, with the same thickness, thus reaffirming that designing this type of concrete shell structures by using optimisation techniques provides an added benefit.

Finally, it is necessary to underline the importance of including in the study a significant phenomenon that affects the shell 
Table 2

Optimisation processes. Final values of objective functions, shell thickness $\left(t_{1}\right)$, maximum compressive stress $\left(\sigma_{c, \text { max }}\right)$ and maximum vertical displacement $\left(U_{z, \text { max }}\right)$.

\begin{tabular}{|c|c|c|c|c|c|c|}
\hline \multirow[t]{2}{*}{ Process } & \multicolumn{3}{|c|}{ Objective functions } & \multirow[t]{2}{*}{$t_{1}(\mathrm{~mm})$} & \multirow[t]{2}{*}{$\sigma_{c, \max }(\mathrm{MPa})$} & \multirow[t]{2}{*}{$U_{z, \max }(\mathrm{mm})$} \\
\hline & $S E(\mathrm{~N} \mathrm{~m})$ & $W(\mathrm{kN})$ & $\sigma_{t}(\mathrm{MPa})$ & & & \\
\hline Initial model & 881.77 & 473.83 & 2.19 & 60.0 & 7.89 & 8.73 \\
\hline$S E\left(t_{\min }=60 \mathrm{~mm}\right)$ & 297.94 & 330.17 & 1.33 & 61.2 & 4.96 & 3.90 \\
\hline$S E\left(t_{\min }=80 \mathrm{~mm}\right)$ & 300.66 & 443.28 & 1.29 & 80.0 & 4.22 & 3.25 \\
\hline$W\left(t_{\min }=60 \mathrm{~mm}\right)$ & 318.29 & 309.54 & 1.26 & 60.0 & 4.89 & 3.94 \\
\hline$W\left(t_{\min }=80 \mathrm{~mm}\right)$ & 367.20 & 431.37 & 1.10 & 80.0 & 4.27 & 3.27 \\
\hline$\sigma_{t}\left(t_{\min }=60 \mathrm{~mm}\right)$ & 514.36 & 767.87 & 1.05 & 159.4 & 4.75 & 2.26 \\
\hline
\end{tabular}

$S E=$ strain energy; $W=$ weight $; \sigma_{t}=$ tensile stress; $t_{\min }=$ minimum thickness.

Table 3

Buckling load (shown as a factor of weight of the shell).

\begin{tabular}{|c|c|c|c|c|c|c|}
\hline & \multirow[t]{2}{*}{ Initial model } & \multicolumn{5}{|c|}{ Optimum design } \\
\hline & & $S E\left(t_{\min }=60\right)$ & $S E\left(t_{\min }=80\right)$ & $W\left(t_{\min }=60\right)$ & $W\left(t_{\min }=80\right)$ & $\sigma_{t}\left(t_{\min }=60\right)$ \\
\hline$t(\mathrm{~mm})$ & 60.0 & 61.2 & 80.0 & 60.0 & 80.0 & 159.4 \\
\hline $\mathrm{L}$ & 8.65 & 17.37 & 25.60 & 15.82 & 22.38 & 53.39 \\
\hline $\mathrm{NL}$ & 5.20 & 12.19 & 16.10 & 11.01 & 14.77 & 22.22 \\
\hline $\mathrm{NL}\left(\alpha_{2}\right)$ & 2.55 & 5.97 & 7.89 & 5.39 & 7.24 & 10.89 \\
\hline
\end{tabular}

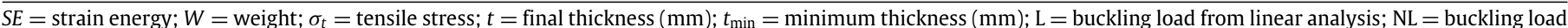
from nonlinear analysis; NL $\left(\alpha_{2}\right)=$ buckling load from nonlinear analysis but modified by a concrete creep factor $\alpha_{2}$.

stability: the structural effect of long-term deformations. The buckling loads of the nonlinear analysis modified by a creep factor are between $66 \%$ and $80 \%$ less than the buckling load of the linear analysis. Nevertheless, this phenomenon has been studied more in depth numerically in [34], in which a reduction of about $10 \%$ in the factor of load-carrying capacity of a shell has been obtained.

\section{Conclusions}

Traditionally, computers have been used, within the process of design of structures, to analyse the response of a user-defined structure and to check its safety for given applied loads. The use of optimisation techniques in the design process of structures widens the field of use of computers and allows the user to obtain optimum designs for stated design conditions.

From the results obtained in the present paper, the following interesting aspects can be highlighted:

(a) Shape optimisation conclusions.

(a1) Form-finding of concrete shells by using shape optimisation techniques leads to considerable improvements in their mechanical behaviour. Slight changes in the shape are usually enough to achieve these improvements.

(a2) Deformations in shells decrease considerably. In the examples studied, final values are less than half the initial values.

(a3) Obtaining the membrane state of compressive stresses in a shell under geometric constraints implies a high cost (a considerable increase of the thickness).

(b) Stability study conclusions.

(b1) The shell structures studied in this work present an excellent behaviour against instability phenomena. The buckling load may be improved by (i) increasing the geometric curvatures; (ii) using a higher elastic modulus of the concrete; and (iii) increasing the shell thickness.

(b2) The behaviour of shells against instability improves noticeably when subjecting them to optimisation. In the examples studied, the buckling loads are approximately double the initial values.

(b3) The buckling load obtained from the nonlinear analysis, modified by the concrete creep factor according to IASS Recommendations, is within a third and a fifth of that obtained in the study of initial stability. This occurs when including a phenomenon that affects the stability: the structural effects of the long-term deformations in concrete.

\section{References}

[1] Ramm E, Kemmler R, Schwarz S. Formfinding and optimization of shell structures. In: Papadrakakis M, Samartín A, Oñate E, editors. Proc 4th int coll computation of shell and spatial structures. ISASR-NTUA. 2000. paper 309.

[2] Ramm E, Wall WA. Shells in advanced computational environment. In: Mang HA, Rammestorfer FG, Eberhardsteiner J, editors. Proc 5th world cong computational mechanics. 2002. Plenary paper.

[3] Manterola J. Relation between structure and shape. ROP 2007;3476:23-40 [in Spanish].

[4] Ohmori H, Yamamoto K. Shape optimization of shell and spatial structures for specified stress distribution-Part 1: Shell analysis. J IASS 1998;39(126):3-13.

[5] Bletzinger KU, Ramm E. Structural optimization and form finding of light weight structures. Comput Struct 2001;79(22-25):2053-62.

[6] Ramm E. Shape finding of concrete shell roofs. J IASS 2004;45(144):29-39.

[7] Ding Y. Shape optimization of structures: A literature survey. Comput Struct 1986;24(6):985-1004.

[8] Gotsis PK. Structural optimization of shell structures. Comput Struct 1994; 50(4):499-507.

[9] Farshad M. Design and analysis of shell structures. Dordrecht: Kluwer Academic Publishers; 1992.

[10] Zingoni A. Shell structures in civil and mechanical engineering. London: Thomas Telford; 1997.

[11] Jawad MH. Design of plate and shell structures. New York: ASME; 2004.

[12] Bendsoe MP, Sigmund O. Topology optimization: Theory, methods and applications. Berlin: Springer-Verlag; 2003.

[13] Arora JS. Introduction to optimum design. London: Elsevier Academic Press; 2004.

[14] ANSYS . Advanced analysis techniques guide. Canonsburg: ANSYS Inc.; 2004.

[15] Hernández S, Fontán AN. Shape and size optimization of three dimensional shells. In: Astudillo R, Madrid AJ, editors. Shells and spatial structures: From recent past to the next millennium. Proc 40th cong IASS. vol. 1. 1999. p. A41-57.

[16] Ramm E, Mehlhorn G. On shape finding methods and ultimate load analyses of reinforced concrete shells. Eng Struct 1991;13:178-98.

[17] Bletzinger KU, Ramm E. Form finding of shells by structural optimization. Eng Comput 1993:9:27-35 [in Germany].

[18] Martí P., Tomás A., Torrano M.S.. The application of optimization techniques in structural concrete teaching. In: García A, editor. Proc 1st ACHE conf structural concrete teaching. ACHE; 2001. p. 203-209 [in Spanish].

[19] Robles SI, Ortega NF. Study of volumetric displacements of shells. J IASS 2001; 42(137):139-47.

[20] Candela F. In defence of the shapeliness and other writings. Madrid: Xarait Libros; 1985 [in Spanish].

[21] Ortega NF, Arias JV. Comparison of mechanical efficiency between an hyperbolic paraboloid and an experimental model. In: Idelsohn S, Oñate E, Dvorkin E, editors. Computational mechanics: New trends and applications. CIMNE. 1998. paper 160.

[22] Muller G, Kabir AF, Scordelis AC. Nonlinear analysis of reinforced concrete hyperbolic paraboloid shells. Mehlhorn G, Ruhle H, Zerna W, editors. Proc IASS symp, vol. 1. Dusseldorf: Werner-Verlag; 1978. p. 191-203.

[23] Domingo A, Lázaro C, Serna P. Design of a thin shell fibre reinforced concrete hypar roof. In: Astudillo R, Madrid AJ, editors. Shells and spatial structures: From recent past to the next millennium. Proc 40th cong IASS. 1999. p. A169-79. 
[24] Scordelis AC. Stability of reinforced concrete domes and hyperbolic paraboloidal shells. In: ACI special publication SP-67. Concrete shell buckling, Farmington Hills: ACI; 1981. p. 63-110.

[25] Basterra A, Chamizo A, Gutiérrez E. Félix Candela and the free edge, the case of Palmira Chapel in Cuernavaca. Bitácora Arquit 2001;5:38-47. [in Spanish].

[26] Csonka P. Deformation and subsequent strengthening of a domical shell in Hungary. Bautechnik 1958;35:69.

[27] Ballesteros P. Nonlinear dynamic buckling of an imperfect spherical concrete shell. In: Mehlhorn G, Ruhle H, Zerna W, editors. Proc IASS symp, vol. 1. Dusseldorf: Werner-Verlag; 1978. p. 205-18.

[28] Medwadowski SJ. Buckling of concrete shells: An overview. J IASS 2004. 45(144):51-63.
29] Tomás A. Optimum design of shape and reinforcement for concrete shells. Ph.D. thesis. Department of Structures and Construction. Cartagena (Spain): Technical University of Cartagena; 2007 [in spanish].

[30] Hofstetter G, Mang HA. Computational mechanics of reinforced concrete structures. Brunswick: Vieweg; 1995.

31] ANSYS. Theory reference. Canonsburg: ANSYS Inc.; 2004

[32] Lee SJ, Hinton E. Dangers inherited in shells optimized with linear assumptions. Comput Struct 2000;78(1-3):473-86.

[33] IASS working group no. 5. Recommendations for reinforced concrete shells and folded plates. adrid: IASS; 1979.

[34] Bockhold J, Petryna YS. Creep influence on buckling resistance of reinforced concrete shells. Comput Struct 2008;86:702-13. 